\title{
The Role of the Dorsomedial Prefrontal Cortex, Basolateral Amygdala, and Dorsal Hippocampus in Contextual Reinstatement of Cocaine Seeking in Rats
}

\author{
Rita A Fuchs*,', K Allison Evans', Christopher C Ledford', Macon P Parker', Jordan M Case', Ritu H Mehta' \\ and Ronald E See' \\ 'Department of Physiology and Neuroscience, Medical University of South Carolina, Charleston, SC, USA
}

\begin{abstract}
The present study tested the hypothesis that separate neural substrates mediate cocaine relapse elicited by drug-associated contextual stimuli vs explicit conditioned stimuli (CSs) and cocaine. Specifically, we investigated the involvement of the dorsal hippocampus (DH), basolateral amygdala (BLA), and dorsomedial prefrontal cortex (dmPFC) in contextual reinstatement of cocaine-seeking behavior and the involvement of the $\mathrm{DH}$ in explicit CS- and cocaine-induced reinstatement. Rats were trained to self-administer cocaine in a distinct context or in the presence of CSs paired explicitly with cocaine infusions. Responding of context-trained rats was then extinguished in the previously cocaine-paired or an alternate context, whereas responding of explicit CS-trained rats was extinguished in the absence of the CSs. Subsequently, the target brain regions or anatomical control regions were functionally inactivated using tetrodotoxin (0 or $5 \mathrm{ng} /$ side), and cocaine-seeking behavior (ie, nonreinforced responses) was assessed in the cocaine-paired context, in the alternate context, in the presence of the explicit CSs, or following cocaine priming ( $10 \mathrm{mg} / \mathrm{kg}$, i.p.). DH inactivation abolished contextual, but failed to alter explicit CS- or cocaine-induced, reinstatement of cocaine-seeking behavior. BLA or dmPFC inactivation also abolished contextual reinstatement. Conversely, inactivation of the control brain regions failed to alter contextual reinstatement. In conclusion, the DH, BLA, and dmPFC play critical roles in contextual reinstatement. Previous findings suggest that the BLA is critical for explicit CS-induced, but not cocaine-primed, reinstatement and the dmPFC is critical for both explicit CS-induced and cocaine-primed reinstatement. Thus, distinct but partially overlapping neural substrates mediate context-induced, explicit CS-induced, and cocaine-primed reinstatement of extinguished cocaine-seeking behavior.

Neuropsychopharmacology (2005) 30, 296-309, advance online publication, 13 October 2004; doi: I 0. I038/sj.npp. I 300579
\end{abstract}

Keywords: cocaine; context; reinstatement; dorsal hippocampus; basolateral amygdala; dorsomedial prefrontal cortex

\section{INTRODUCTION}

Relapse is a major impediment in the treatment of cocaine dependence. Drug-associated environments, discrete conditioned stimuli (CSs, eg, paraphernalia), and exposure to cocaine elicit craving and relapse in drug users (Jaffe $e t$ al, 1989; Ehrman et al, 1992; Foltin and Haney, 2000). Similarly, cocaine-paired contextual stimuli (Rauhut et al, 2000; Alleweireldt et al, 2001; Crombag et al, 2002), explicit CSs (de Wit and Stewart, 1981), and cocaine priming injections (Gerber and Stretch, 1975) elicit cocaine-seeking behavior, an index of incentive motivation for cocaine in rats.

*Correspondence: Dr RA Fuchs, Department of Physiology and Neuroscience, Medical University of South Carolina, 173 Ashley Avenue, Suite 403, Charleston, SC 29425, USA, Tel: + I 8430792 6333, Fax: + I 843792 4423, E-mail: fuchs@musc.edu

Received 15 July 2003; revised I I August 2004; accepted 23 August 2004

Online publication: 9 September 2004 at http://www.acnp.org/citations/ Npp090 I0404327/default.pdf
Cocaine-seeking behavior elicited by drug-paired contextual stimuli, explicit CSs, and cocaine appears to be identical; however, different cognitive mechanisms likely mediate the motivational effects of these stimuli. During drug self-administration training, explicit CSs are presented response contingently and consistently paired with a drug reinforcer. Thus, explicit CSs acquire the ability to elicit drug seeking by signaling imminent drug reinforcement or by acting as conditioned reinforcers. In contrast, contexts are comprised of static multimodal background stimuli, which constitute a setting where response-drug and/or CS-drug associations form. Consequently, contexts elicit drug seeking primarily by functioning as 'occasion setters,' discriminative stimuli $\left(\mathrm{S}_{\mathrm{D}}\right)$ which signal responsecontingent drug availability (Gordon and Klein, 1994). Lastly, cocaine is thought to produce drug seeking by directly stimulating the brain reward system (for review see, Shalev et al, 2002).

Given the putative differences in the informational content of drug-paired contexts, explicit CSs, and cocaine, separate brain systems likely mediate their motivational 
effects. Previous research indicates that the dorsomedial prefrontal cortex (dmPFC), basolateral amygdala (BLA), and hippocampus exhibit neuronal activation concomitant with cocaine-seeking behavior in a cocaine-paired environment (Neisewander et al, 2000), and lesions of these structures impair place conditioning, a task dependent on context-reward learning (Tzschentke and Schmidt, 1999; Ferbinteanu and McDonald, 2001; Fuchs et al, 2002b; Meyers et al, 2003). BLA or dmPFC inactivation also impairs explicit CS-induced reinstatement of cocaine seeking (McLaughlin and See, 2003), a behavior based on explicit CS-reward associations (Kruzich and See, 2001). Furthermore, dmPFC inactivation impairs, whereas BLA inactivation fails to alter, cocaine-primed reinstatement of cocaine-seeking behavior (McFarland and Kalivas, 2001). Unlike the BLA and dmPFC, the dorsal hippocampus (DH) does not play a critical role in explicit CS-US associations according to the fear conditioning literature (Kim and Fanselow, 1992); however, its involvement in explicit CSinduced and cocaine-primed reinstatement of cocaineseeking behavior has yet to be examined. Importantly, it remains to be investigated whether the functional integrity of these structures is necessary for contextual reinstatement of cocaine-seeking behavior.

The primary objective of the present study was to test the hypothesis that distinct, albeit likely overlapping, neural circuitries mediate the ability of contextual $\mathrm{S}_{\mathrm{D}} \mathrm{s} v s$ explicit CSs and cocaine to elicit cocaine-seeking behavior. To this end, following drug self-administration training and extinction, the dmPFC, BLA, or DH were functionally inactivated using the sodium channel blocker, tetrodotoxin, and resulting alterations in cocaine-seeking behavior were assessed in a cocaine-paired context or a non-cocainepaired, alternate context. Effects of $\mathrm{DH}$ inactivation on explicit CS- and cocaine-induced reinstatement were also assessed. It was postulated that BLA or dmPFC inactivation would impair contextual reinstatement of cocaine-seeking behavior and $\mathrm{DH}$ inactivation would selectively impair contextual, but not explicit CS- or cocaine-induced, reinstatement.

\section{MATERIALS AND METHODS}

\section{Animals}

Male Sprague-Dawley rats (Charles-River, $N=87$ ), weighing $300-325 \mathrm{~g}$ at the start of the experiment, were individually housed in a temperature and humidity controlled vivarium on a reversed light-dark cycle. Rats were maintained on 20-25 g of rat chow per day, with water available ad libitum. The housing and treatment of the rats followed the guidelines of the 'Guide for the Care and Use of Laboratory Rats' (Institute of Laboratory Animal Resources on Life Sciences, National Research Council, 1996). Rats were given 5 days for adaptation before the start of the experiment.

\section{Food Training}

Rats were trained to lever press on a fixed ratio (FR) 1 schedule of food reinforcement ( $45 \mathrm{mg}$ pellets; Noyes, Lancaster, $\mathrm{NH}$ ) in sound-attenuated operant conditioning chambers $(30 \times 20 \times 24 \mathrm{~cm}$ high; Med Associates Inc., St Albans, VT) during a 16-h overnight food training session. The chambers were equipped with two retractable levers, a stimulus light above each lever, a food pellet dispenser between the levers, a house light on the wall opposite to the levers, and a speaker connected to a tone generator (ANL926, Med Associates). During the session, the stimuli subsequently used for contextual or explicit cue conditioning were not presented. In the standard operant conditioning chamber, each lever press on the active lever resulted in delivery of a food pellet only. Lever presses on the inactive lever had no programmed consequences. Following food training, food pellet dispensers were removed from the chambers.

\section{Surgery}

At $48 \mathrm{~h}$ after food training, rats were anesthetized using ketamine hydrochloride $(66.6 \mathrm{mg} / \mathrm{kg}$, IP; Fort Dodge Animal Health, Fort Dodge, IA) and xylazine (1.3 mg/kg, IP; Bayer, Shawnee Mission, KS) followed by equithesin $(1 \mathrm{ml} / \mathrm{kg}$ of a solution of $9.72 \mathrm{mg} / \mathrm{ml}$ pentobarbital sodium, $42.5 \mathrm{mg} / \mathrm{ml}$ chloral hydrate, and $21.3 \mathrm{mg} / \mathrm{ml}$ magnesium sulfate heptahydrate dissolved in a $44 \%$ propylene glycol, $10 \%$ ethanol solution; IP). Chronic indwelling catheters were constructed using a bent steel cannula with a screw-type connector (Plastics One, Roanoke, VA), SILASTIC tubing $(10 \mathrm{~cm}$, i.d. $0.64 \mathrm{~mm}$, o.d. $1.19 \mathrm{~mm}$, Dow Corning, Midland, MI), Marlex mesh, and cranioplastic cement, as described previously (See et al, 2001). The end of the catheter was inserted into the right jugular vein and was secured to surrounding tissue with suture. The catheter ran subcutaneously and exited on the rat's back, posterior to the shoulder blades.

Immediately after the catheter surgery, the rats were placed into a stereotaxic instrument (Stoelting, Wood Dale, IL). They were implanted with bilateral stainless-steel guide cannulae (26 gauge, Plastics One) aimed at the BLA, dmPFC, DH or their respective cortical anatomical control brain regions, the barrel field region of the somatosensory cortex (SSbf), the ventromedial prefrontal cortex (vmPFC), or the trunk region of the somatosensory cortex (SStr), using standard stereotaxic procedures (BLA: $-2.5 \mathrm{~mm} \mathrm{AP}$, $\pm 5.0 \mathrm{~mm} \mathrm{ML},-6.6 \mathrm{~mm} \mathrm{DV}$; dmPFC: $+3.0 \mathrm{~mm} \mathrm{AP}$, $\pm 0.6 \mathrm{~mm} \mathrm{ML},-1.4 \mathrm{~mm} \mathrm{DV}$; DH: cannulae angled at $15^{\circ}$ : $-3.1 \mathrm{~mm} \mathrm{AP}, \pm 3.1 \mathrm{~mm} \mathrm{ML},-2.1 \mathrm{~mm} \mathrm{DV}$; SSbf: $-2.5 \mathrm{~mm}$ $\mathrm{AP}, \pm 5.0 \mathrm{~mm} \mathrm{ML},-2.3 \mathrm{~mm} \mathrm{DV}$; vmPFC: $+3.0 \mathrm{~mm} \mathrm{AP}$, $\pm 0.6 \mathrm{~mm} \mathrm{ML},-4.2 \mathrm{~mm} \mathrm{DV}$; SStr, cannulae angled at $15^{\circ}$ : $-3.1 \mathrm{~mm} \mathrm{AP,} \pm 3.1 \mathrm{~mm} \mathrm{ML},-1.0 \mathrm{~mm} \mathrm{DV}$, relative to bregma). These anatomical control brain regions are in the same anterior-posterior and medial-lateral planes as the target brain regions and are adjacent to the target brain regions with the exception of the SSbf. The SSbf was chosen over more ventral brain regions as the anatomical control region for the BLA in order to minimize the inactivation of the external capsule. Three small screws and cranioplastic cement secured the guide cannulae to the skull. Stylets (Plastics One) were placed into the guide cannulae and catheter to prevent occlusion. To extend catheter patency, the catheters were flushed twice daily for 5 days following surgery with $0.1 \mathrm{ml}$ of an antibiotic solution of cefazolin (10.0 mg/ml, Schein Pharmaceutical, Florham Park, NJ) dissolved in heparinized saline $(70 \mathrm{U} / \mathrm{ml}$; Elkins-Sinn, 
Cherry Hill, NJ). Thereafter, catheters were flushed with $0.1 \mathrm{ml}$ heparinized saline $(10 \mathrm{U} / \mathrm{ml})$ prior to each selfadministration session, and with $0.1 \mathrm{ml}$ of the cefazolin solution and $0.1 \mathrm{ml}$ of heparinized saline $(70 \mathrm{U} / \mathrm{ml})$ after each session. Catheter patency was periodically verified by infusing $0.08-0.10 \mathrm{ml}$ of methohexital sodium $(20 \mathrm{mg} / \mathrm{ml}$, IV; Eli Lilly and Co., Indianapolis, IN), which produces a rapid loss of muscle tone only when administered intravenously.

\section{Self-Administration}

Self-administration training was conducted during 2 -h sessions on 10 consecutive days during the rats' dark cycle. Rats were trained to press a lever according to an FR 1 schedule of cocaine reinforcement (cocaine hydrochloride; $0.20 \mathrm{mg} / 0.05 \mathrm{ml}$; National Institute on Drug Abuse, Research Triangle Park, NC) with a 40-s time out period following each reinforcer. The catheters were connected to liquid swivels (Instech, Plymouth Meeting, PA) via polyethylene 20 tubing (Dow Corning Co., Midland, MI) that was encased in steel spring leashes (Plastics One). The swivels were suspended above the operant conditioning chamber and were connected to infusion pumps (Model PHM-100, Med Associates) with Tygon tubing (Performance Plastics, Akron, $\mathrm{OH}$ ). Data collection and reinforcer delivery were controlled using Schedule Manager for Windows software version 2.09 (Med Associates).

\section{Intracranial Infusions}

For intracranial infusions, stainless-steel injection cannulae (33 gauge, Plastics One) were inserted to a depth of $1 \mathrm{~mm}$ (dmPFC, DH, vmPFC, SStr) or $2 \mathrm{~mm}$ (BLA, SSbf) below the tip of the guide cannulae immediately prior to placement into the chamber. The injection cannulae were connected to 10- $\mu$ l Hamilton syringes (Hamilton Co., Reno, NV) that were mounted on an infusion pump (Harvard Apparatus, South Natick, MA). TTX (5.0 ng/side) or phosphatebuffered saline vehicle $(\mathrm{pH}=7.0$ for both) were infused bilaterally at a volume of $0.5 \mu \mathrm{l} /$ hemisphere over $2 \mathrm{~min}$. The injection cannulae were left in place for $1 \mathrm{~min}$ prior to and after the infusion. TTX acts by inhibiting the generation and propagation of action potentials (for a critical review of this and other temporary inactivation procedures, see Martin and Ghez, 1999). Metabolic activation and other physiological studies have shown that the mean inactivated tissue radius after infusion of $5 \mathrm{ng} / 0.5 \mu \mathrm{l}$ of TTX is $\sim 0.7-0.8 \mathrm{~mm}$, and the maximal effect of TTX lasts for no less than $120 \mathrm{~min}$, then disappears exponentially within $24 \mathrm{~h}$ (Cahill et al, 1987; Zhuravin and Bures, 1991; Ambrogi Lorenzini et al, 1995). Thus, subjects can be used as their own controls. Using an infusion protocol and coordinates identical to those utilized in the present experiments, we have been able to establish functional dissociation between the dmPFC and vmPFC (McLaughlin and See, 2003), as well as between the BLA and the SSbf (McLaughlin and See, 2003) and central nucleus of the amygdala (Kruzich and See, 2001), suggesting that TTX administration resulted in selective inactivation of the target structures in the present study.

\section{Experiment 1: Effects of BLA, dmPFC, DH, SSbf, vmPFC, or SStr Inactivation on Contextual Reinstatement of Cocaine-Seeking Behavior}

Previous studies using the renewal procedure have demonstrated that Pavlovian and operant conditioned responses to an explicit CS can be reinstated when a subject is returned to the conditioning context after extinction in another context, suggesting that context acts as an occasion setter (Bouton and Bolles, 1979; Bouton and Swartzentruber, 1986; Bouton and Ricker, 1994; Goddard, 1999; Nakajima et al, 2000). Similarly, it has been shown that re-exposure to a drug-associated context is sufficient to reinstate drugseeking behavior in the response-contingent presence (Crombag et al, 2002; Crombag and Shaham, 2002) or absence (Rauhut et al, 2000; Alleweireldt et al, 2001) of a drug-paired explicit CS. However, the neural substrates that mediate context-induced reinstatement of drug seeking have not been identified. Thus, Experiment 1 examined the involvement of the BLA, dmPFC, and DH in contextinduced reinstatement of cocaine seeking.

Stimulus presentation during self-administration training. Rats $(N=53)$ were trained to self-administer cocaine in operant conditioning chambers during 2 -h daily sessions. The chambers contained one of two distinctly different sets of visual, auditory, olfactory, and tactile contextual stimuli (see Table 1). The contextual stimuli were presented throughout each session independent of responding. Active lever presses resulted in a 2-s activation of the infusion pump only. After each infusion, responses on the active lever were counted but had no consequences during the 40-s time-out period. During the sessions, responses on the inactive lever had no programmed consequences, but were recorded.

Extinction. Rats underwent 2-h extinction sessions on at least 10 consecutive days, during which responses on either lever had no programmed consequences. Extinction sessions were conducted either in the self-administration

Table I Contextual Stimuli Used in Experiment I

Stimulus components

\begin{tabular}{lllll}
\cline { 2 - 4 } Contexts & Visual & Auditory & Olfactory & Tactile \\
\hline I & Black floor board, red house light & Clicking sound $(78 \mathrm{~dB}, 10 \mathrm{~Hz})$ & Vanilla scented oil & Rough-textured floor board \\
2 & Clear floor board, flashing stimulus light above inactive lever & Pure tone $(78 \mathrm{~dB}, 2 \mathrm{kHz})$ & Sandalwood scented oil & Smooth floor board \\
\hline
\end{tabular}

Neuropsychopharmacology 
Table 2 Contextual Stimulus Presentation During the Three Phases of Experiment I

Experimental phases

\begin{tabular}{|c|c|c|c|c|c|}
\hline \multirow[b]{2}{*}{ Groups } & \\
\hline & Self-administration & & Extinction & & Reinstatement \\
\hline \multirow[t]{3}{*}{ Cocaine context } & Context I (cocaine-paired) & $\rightarrow$ & Context 2 (alternate) & $\rightarrow$ & Context I (cocaine-paired) \\
\hline & Context 2 (cocaine-paired) & $\rightarrow$ & Context I (alternate) & $\rightarrow$ & Context 2 (cocaine-paired) \\
\hline & & $\rightarrow$ & & $\rightarrow$ & \\
\hline \multirow[t]{2}{*}{ Alternate context } & Context I (cocaine-paired) & $\rightarrow$ & Context I (cocaine-paired) & $\rightarrow$ & Context 2 (alternate) \\
\hline & Context 2 (cocaine-paired) & $\rightarrow$ & Context 2 (cocaine-paired) & $\rightarrow$ & Context I (alternate) \\
\hline
\end{tabular}

context (alternate context group; $N=27$ ) or in the alternate context (cocaine context group; $N=52$, see Table 2). Assignment of the BLA-, dmPFC-, and DH-cannulated rats to the alternate and cocaine context groups was counterbalanced based on cocaine intake during selfadministration, whereas in the follow-up experiments, all SSbf-, vmPFC-, and SStr-cannulated rats received extinction training in the alternate context. To examine the effects of the intracranial infusion procedure on responding, rats received a sham intracranial infusion prior to placement into the chamber on extinction day 7. During the sham procedure, injection cannulae were bilaterally inserted into the rat's guide cannulae and were left in place for $4 \mathrm{~min}$, but fluid was not infused through the infusion cannulae. Extinction sessions were terminated when the rats reached the criterion for extinction (ie, $\leqslant 25$ active lever responses/ session on 2 consecutive days).

Contextual reinstatement test. On the test days, rats received bilateral intracranial microinfusions of TTX (5.0 ng/ $0.5 \mu \mathrm{l} /$ side). or phosphate-buffered saline vehicle $(0.5 \mu \mathrm{l} /$ side $)$ over $2 \mathrm{~min}$ immediately before reinstatement testing. Rats that had received microinfusions into the BLA, dmPFC, or DH were then placed into the self-administration context (cocaine context group) or an alternate context (alternate context group, see Table 2) for a 2-h test session. All rats that had received microinfusions into the anatomical control brain regions (ie SSbf, vmPFC, or SStr) were placed into the self-administration context on the reinstatement test days. This design resulted in the following groups: BLA cocaine context $(N=9)$, BLA alternate context $(N=9)$, dmPFC cocaine context $(N=7)$, dmPFC alternate context $(N=10), \mathrm{DH}$ cocaine context $(N=10), \mathrm{DH}$ alternate context $(N=8)$, SSbf cocaine context $(N=8)$, vmPFC cocaine context $(N=10)$, SStr cocaine context $(N=8)$. The order of TTX and vehicle infusions was counterbalanced across two test sessions based on the history of cocaine intake during self-administration. During testing, lever presses were recorded but had no scheduled consequences. Between the two test sessions, the rats underwent additional extinction sessions in the self-administration context (alternate context group) or the alternate context (cocaine context group) until they reached the criterion for extinction described above.

Locomotor activity test. Studies from our laboratory (McLaughlin and See, 2003) have demonstrated that TTX microinfusions into the BLA do not significantly alter general activity during a 1-h locomotor activity test, suggesting that general motor deficits do not account for the effects of BLA inactivation on reinstatement. To further assess possible motor impairing effects of intracranial TTX infusions, we examined the effects of TTX infused into the dmPFC, DH, vmPFC, SStr, or SSbf on general activity.

Four days following the last reinstatement test, locomotor activity was measured in novel Plexiglas chambers $(40.8 \mathrm{~cm}$ $W \times 40.8 \mathrm{~cm} L \times 37.5 \mathrm{~cm} H$ ) equipped with an array of eight photodetectors and corresponding light sources that emitted photobeams $8 \mathrm{~cm}$ apart at $4.5 \mathrm{~cm}$ above the chamber floor. A computerized activity system (San Diego Instruments, San Diego, CA) recorded the number of times neighboring photobeams were broken consecutively by a rat moving in the chamber during a 1 -h test session. Prior to placement into the apparatus, rats received bilateral intracranial infusions of TTX or vehicle into the dmPFC, $\mathrm{DH}$, vmPFC, SStr, or SSbf using the procedures described above. Assignment to TTX vs vehicle infusions was randomized. Data from nine additional rats with correct cannula placement (vmPFC, $N=2$; SSbf, $N=4$; SStr, $N=3$ ) was included only in the locomotion study because of technical problems that disrupted contextual conditioning. The resulting group sizes for the locomotor study were as follows: dmPFC vehicle $(N=5)$, dmPFC TTX $(N=5), \mathrm{DH}$ vehicle $(N=6)$, DH TTX $(N=5)$, vmPFC vehicle $(N=6)$, vmPFC TTX $(N=6)$, SSbf vehicle $(N=6)$, SSbf TTX $(N=6)$, SStr vehicle $(N=6)$, and SStr TTX $(N=6)$.

\section{Experiment 2: Effects of Dorsal Hippocampus Inactivation on Explicit CS-Induced and Cocaine-Primed Reinstatement of Cocaine Seeking}

Previous research from our laboratory has demonstrated that inactivation of the dmPFC or BLA impairs explicit CSinduced reinstatement of extinguished cocaine-seeking behavior (McLaughlin and See, 2003). Furthermore, inactivation of the dmPFC, but not of the BLA, abolishes cocaine-primed reinstatement of extinguished cocaineseeking behavior (McFarland and Kalivas, 2001). It has not been examined, however, whether the functional integrity of the $\mathrm{DH}$ is necessary for explicit CS-induced or cocaine-induced relapse to cocaine seeking. In order to facilitate comparison of the neural circuitries that mediate various forms of relapse to drug seeking, Experiment 2 examined the effects of $\mathrm{DH}$ inactivation on explicit 
CS-induced and cocaine-primed reinstatement of extinguished cocaine-seeking behavior.

Stimulus presentation during self-administration training. Rats $(N=8)$ were trained to self-administer cocaine in standard operant conditioning chambers during 2-h daily sessions. The house light was illuminated throughout each session. Lever presses on the active lever resulted in a 2-s activation of the infusion pump and a 5-s presentation of a CS complex, consisting of activation of the white stimulus light above the active lever and the tone generator $(78 \mathrm{~dB}$, $2 \mathrm{kHz}$ ). After each infusion, responses on the active lever were counted but had no consequences during the 40-s time-out period. During the sessions, responses on the inactive lever were recorded but had no programmed consequences.

Extinction. Rats underwent 2-h extinction sessions on at least 10 consecutive days, during which responses on either lever were recorded but had no programmed consequences. Extinction sessions were terminated when the rats reached the criterion for extinction (ie, $\leqslant 25$ active lever responses/ session on 2 consecutive days). To examine the effects of the intracranial infusion procedure on responding, rats received a sham intracranial infusion prior to placement into the chamber on extinction day 7 , using the sham procedure described above.

Explicit CS-induced reinstatement test. Two reinstatement tests were conducted to examine the effects of $\mathrm{DH}$ inactivation on the ability of the light-tone CS complex to reinstate responding. On the test days, rats first received infusions of TTX or vehicle into the DH. Rats were then placed into the chambers for a 2 -h session, during which responses resulted in 5-s presentations of the light-tone CSs in the absence of cocaine reinforcement. Each CS presentation was followed by a 40 -s time-out period during which responses had no programmed consequences. The order of TTX $v s$ vehicle infusions was counterbalanced across the two test days based on cocaine intake. Prior to the second test day, rats underwent additional extinction sessions until they exhibited $\leqslant 25$ active lever responses per session on 2 consecutive days.

Cocaine-primed reinstatement test. Following the second explicit CS-induced reinstatement test, rats underwent additional extinction sessions until they reached the extinction criterion described above. Subsequently, two tests were conducted to examine the effects of $\mathrm{DH}$ inactivation on the ability of a cocaine priming injection to reinstate responding. On the test days, rats first received TTX or vehicle infusions into the $\mathrm{DH}$ and a cocaine priming injection $(10 \mathrm{mg} / \mathrm{kg}$, i.p.). Rats were then placed into the chambers for a 2-h extinction session, during which the light-tone CSs were not presented and responses had no programmed consequences. The $10 \mathrm{mg} / \mathrm{kg}$ priming dose of cocaine was selected based on previous studies showing that it produces maximal reinstatement of cocaine seeking (Cornish et al, 1999). The order of TTX $v s$ vehicle infusions was counterbalanced across the two test days based on cocaine intake, and was either the same or reversed as that during the explicit CS-induced reinstatement tests. Prior to the second cocaine reinstatement test day, rats underwent additional extinction sessions until they reached the extinction criterion.

Histology. Rats were transcardially perfused with phosphate-buffered saline and $10 \%$ formaldehyde solution. The brains were dissected out and were stored in $10 \%$ formaldehyde solution until sectioning. Brains were sectioned in the coronal plane at a thickness of $75 \mu \mathrm{m}$ on a vibratome (Technical Products International, Inc., St Louis, $\mathrm{MO})$. Sections containing cannula tracks were mounted on gelatin-coated slides and were stained with cresyl violet (Kodak, Rochester, NY). The sections were examined under a light microscope to determine cannula placement. The most ventral point of each cannula track was mapped onto schematics of the appropriate plates from the rat brain atlas of Paxinos and Watson (1997).

Data analysis. Mixed-factor and repeated measures analyses of variance (ANOVA) were used to analyze responses on the active and inactive levers with lever (active, inactive) and day (self-administration, extinction, TTX reinstatement day, vehicle reinstatement day) as within-subjects factors, and group (alternate context group, cocaine-paired context group) as a between-subjects factor, where appropriate. Statistically significant interaction effects were further investigated using Tukey HSD post hoc tests. Planned one-tail $t$-tests were used to compare responding during the explicit CS- and cocaine-induced reinstatement tests to responding during self-administration training and to compare responding during the cocaine-induced reinstatement tests to the extinction baseline.

\section{RESULTS}

\section{Histology}

Representative photomicrographs and/or schematics illustrating the distribution of injection cannula placements in the brains of rats from Experiments 1 and 2 are included in Figures 1 and 2, respectively. The most ventral point of the injection cannula tracks was located within the target region bilaterally for all rats whose data were included in data analysis and in the Ns reported above. The target regions were defined as follows: $d m P F C$, the $\mathrm{Cg} 1$ region and the $\mathrm{Cg}$ $1 / \mathrm{Cg} 2$ transition area of the medial prefrontal cortex; $v m P F C$, the ventral $\mathrm{Cg} 2$, ventromedial orbitofrontal, and infralimbic areas of the ventromedial prefrontal cortex, $B L A$, the lateral/basolateral nuclei of the amygdala; $S S b f$, the barrel fields region of the somatosensory cortex that overlies the basolateral amygdala; $D H$, the dorsal hippocampus; SStr, the trunk region of the somatosensory cortex that overlies the dorsal hippocampus. The vmPFC, SSbf, and SStr were selected as anatomical controls regions because they are either adjacent and/or dorsal (ie, in the path of TTX diffusion) relative to the brain regions of primary interest (ie, dmPFC, BLA, and $\mathrm{DH}$, respectively). Highpower microscopy of the infused area did not reveal any abnormalities in neuronal integrity. 

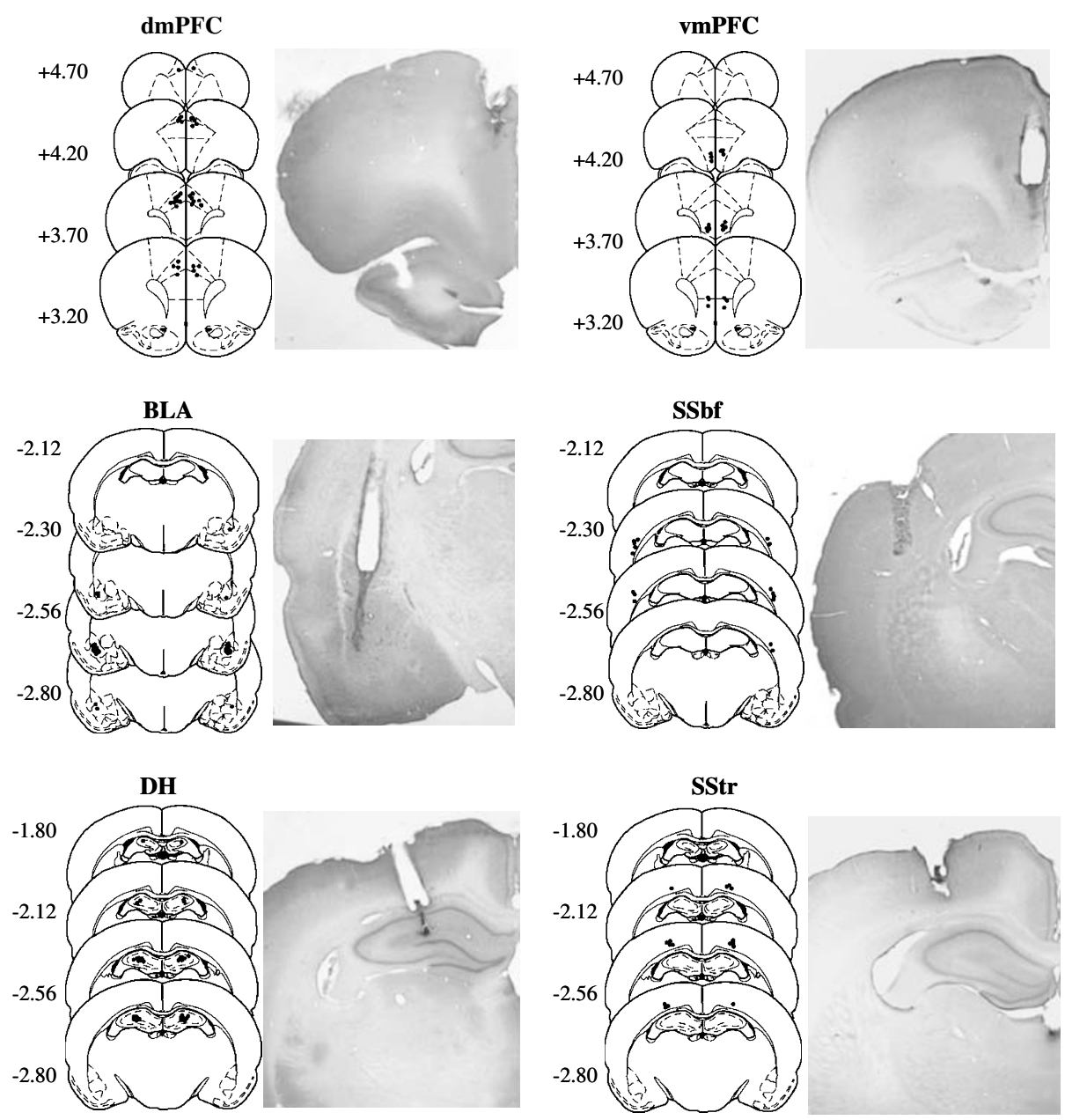

Figure I Microinfusion cannula placement as verified on cresyl violet-stained sections and representative photomicrographs of each brain region of interest (left) and its respective anatomical control brain region (right) in Experiment I. The symbols represent the most ventral point of the infusion cannula tract for each rat on coronal sections based on the atlas of Paxinos and Watson (1997). Numbers indicate the distance from bregma in millimeters.

\section{Experiment 1}

Self-administration and extinction. Rats exhibited stable responding on the active lever during the last 7 selfadministration days with a within-subject variability of less than $10 \%$ in daily cocaine intake. There was no difference in cocaine intake between the groups with guide cannulae aimed at the dmPFC, BLA, DH, vmPFC, SSbf, or SStr $\left(\mathrm{F}_{(5,73)}=0.46, P=0.80\right)$. The mean daily cocaine intake $( \pm$ SEM) was $31.81 \pm 1.3$ infusions (approx. $20.05 \pm 1.10 \mathrm{mg} /$ $\mathrm{kg} /$ session).

Responding declined upon removal of cocaine reinforcement and extinguished to criterion $(\leqslant 25$ responses/day per two consecutive days) prior to reinstatement testing (Figure 3) independent of the context in which extinction training occurred (data not shown). The effects of context on extinction training were examined in DH-, BLA-, and dmPFC-cannulated rats. SStr-, SSbf-, or vmPFC-cannulated rats were not included in these analyses because none of these subjects underwent extinction training in the previously cocaine-paired context. Separate $2 \times 7$ mixed factor ANOVAs of active lever presses indicated that responding declined across extinction sessions $\left(\mathrm{F}_{(6,90-96)}=15.00-16.87\right.$,
$P<0.0001)$. Extinction responding was not significantly altered by whether the rats underwent extinction training in the previously cocaine-paired or in the alternate context (context main effect: $\mathrm{F}_{(1,15-16)}=0.31-1.47, P=0.59-0.24$; context by day interaction effect: $\mathrm{F}_{(6,90-96)}=0.85-1.35$, $P=0.53-0.24)$. There was also no effect of context on the total number of days needed to reach the extinction criterion prior to the first or second reinstatement tests $\left(t_{(15-16)}=0.284-1.865, P=0.08-0.78\right)$. The mean number of days $( \pm$ SEM) needed for rats to reach the extinction criterion was $11.58 \pm 0.38$ and $2.68 \pm 0.23$ days prior to the first and second reinstatement test days, respectively. Furthermore, sham intracranial infusions on extinction day 7 did not alter extinguished responding (data not shown).

Contextual reinstatement of extinguished cocaine-seeking behavior. Exposure to the cocaine-paired context reinstated extinguished cocaine-seeking behavior, whereas exposure to the alternate context failed to reinstate cocaine-seeking behavior. Furthermore, TTX inactivation of the BLA, dmPFC, or DH disrupted the ability of the cocaine-paired 


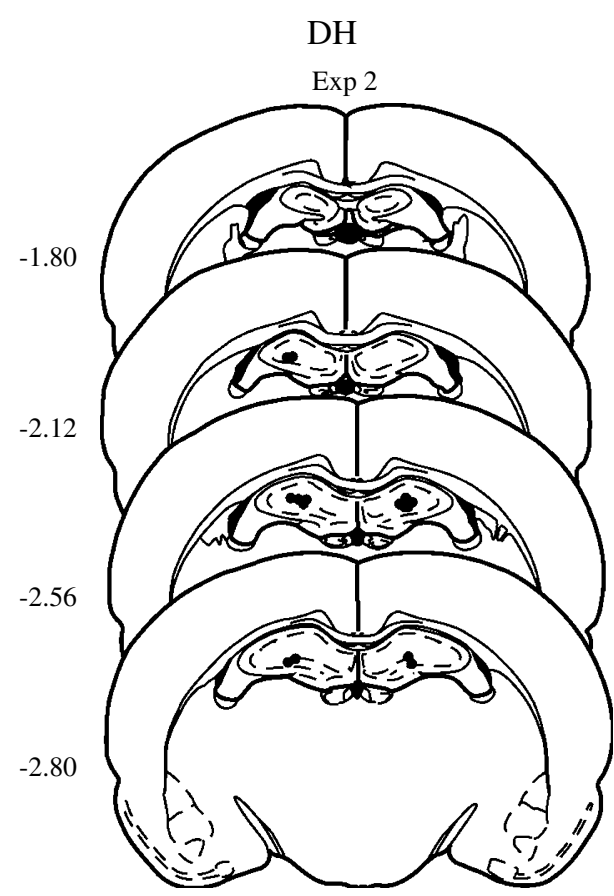

Figure 2 Microinfusion cannula placement within the $\mathrm{DH}$ as verified on cresyl violet-stained sections in Experiment 2. The symbols represent the most ventral point of the infusion cannula tract for each rat on coronal sections based on the atlas of Paxinos and Watson (1997). Numbers indicate the distance from bregma in millimeters.

context to reinstate extinguished cocaine-seeking behavior (Figure 3 left), whereas inactivation of the adjacent SSbf, vmPFC, or SStr failed to alter responding (Figure 3 right). There was no difference in lever responding on the extinction days that immediately preceding the TTX and vehicle reinstatement test days; therefore, extinction data were collapsed across these time points (extinction, henceforth)

For the BLA, dmPFC, and DH, separate $3 \times 2 \times 2$ mixed factor ANOVAs of lever presses indicated statistically significant three-way day by group by lever interaction effects $\left(\mathrm{BLA}, \mathrm{F}_{(2,32)}=5.46, P<0.01 ; \mathrm{dmPFC}, \mathrm{F}_{(2,30)}=6.37\right.$, $P<0.005$; DH, $\left.\mathrm{F}_{(2,32)}=7.62, P<0.005\right)$. Subsequent pairwise comparisons revealed that in vehicle-pretreated rats, exposure to the cocaine-paired context produced a significant increase in responding on the active lever relative to extinction (Tukey test, $P<0.05$ ) and relative to the inactive lever (Tukey test, $P<0.05$ ). Furthermore, exposure to the cocaine-paired context elicited more responding on the active lever relative to exposure to the alternate context (Tukey test, $P<0.05$ ). Exposure to the alternate context failed to elicit an increase in responding on the active lever relative to the extinction baseline or to responding on the inactive lever. Pretreatment with TTX into the BLA, dmPFC, or DH blocked reinstatement of responding in the cocainepaired context (Tukey test, $P<0.05$ ), whereas it did not alter responding in the alternate context. Pairwise comparisons of responses on the inactive lever did not reveal any significant day or group effects.

For the SSbf and VmPFC, separate $3 \times 2$ mixed factor ANOVAs of lever presses indicated significant main effects of day (SSbf: $\mathrm{F}_{(2,14)}=5.66, P<0.05$; vmPFC, $\mathrm{F}_{(2,18)}=4.09$, $P<0.5)$ and lever (SSbf: $\mathrm{F}_{(1,7)}=21.01, P<0.005$; vmPFC, $\left.\mathrm{F}_{(1,9)}=12.68, P<0.01\right)$, but no interaction effects. However, planned comparisons indicated that exposure to the cocaine-paired context produced a significant increase in responding on the active lever relative to extinction (SSbf: $t_{(7)}=3.54, P<0.01$; vmPFC, $\left.t_{(9)}=2.26, P=0.05\right)$. Furthermore, pretreatment with TTX into the SSbf or vmPFC failed to alter reinstatement of responding in the cocaine-paired context relative to saline pretreatment (SSbf: $t_{(7)}=0.25$, $P=0.81$; vmPFC, $\left.t_{(9)}=0.15, P=0.88\right)$. Planned comparisons of responses on the inactive lever did not reveal any significant day effects. For the SStr, the $3 \times 2$ mixed factor ANOVA of lever presses indicated a statistically significant two-way day by lever interaction effect $\left(\mathrm{SStr} \mathrm{F}_{(2,14)}=1.58\right.$, $P<0.05)$. Subsequent pairwise comparisons revealed that in vehicle-pretreated rats, exposure to the cocaine-paired context produced a significant increase in responding on the active lever relative to extinction (Tukey test, $P<0.05$ ) and relative to the inactive lever (Tukey test, $P<0.05$ ). However, pretreatment with TTX into the SStr failed to alter the reinstatement of responding in the cocaine-paired context (Tukey test, $P>0.05$ ). Pairwise comparisons of responses on the inactive lever did not reveal any significant day effects.

\section{Experiment 2}

Self-administration and extinction. Similar to Experiment 1 , rats exhibited stable responding on the active lever during the last 7 self-administration days with a withinsubject variability of less than $10 \%$ in daily cocaine intake. The mean daily cocaine intake $( \pm$ SEM) was $32.63 \pm 2.21$ infusions (approx. 20.88 $\pm 1.42 \mathrm{mg} / \mathrm{kg} / \mathrm{session}$ ). There was no difference in cocaine intake between the explicit cuetrained rats of Experiment 2 and the DH group of Experiment $1\left(t_{(24)}=0.21, P>0.5\right)$. Responding declined upon removal of cocaine reinforcement $\left(\mathrm{F}_{(6,42)}=9.01\right.$, $P<0.0001$; Tukey test, $P<0.05)$ and extinguished to criterion prior to reinstatement testing (Figure 4). Furthermore, sham infusions into the $\mathrm{DH}$ on extinction day 7 did not reinstate extinguished responding.

Explicit cue-induced reinstatement of extinguished cocaine-seeking behavior. Response-contingent presentation of the light-tone CSs elicited robust reinstatement of responding on the active lever (Figure 4). Furthermore, a comparison of active lever responding exhibited by rats that received vehicle infusions into the DH in Experiments 1 and 2 revealed that the time course of lever pressing was similar in response to the light-tone CSs $v s$ the cocaine-paired context, even though response-contingent presentation of the light-tone CSs elicited more responding overall (data not shown).

TTX inactivation of the DH failed to alter the ability of the light-tone CSs to reinstate responding (Figure 4a). There was no significant difference in responding during the extinction days that immediately preceded the vehicle and TTX reinstatement test days; therefore, extinction data were collapsed across these time points (extinction, henceforth). The $3 \times 2$ repeated measures ANOVA of lever presses during the reinstatement test days and extinction indicated 
dmPFC
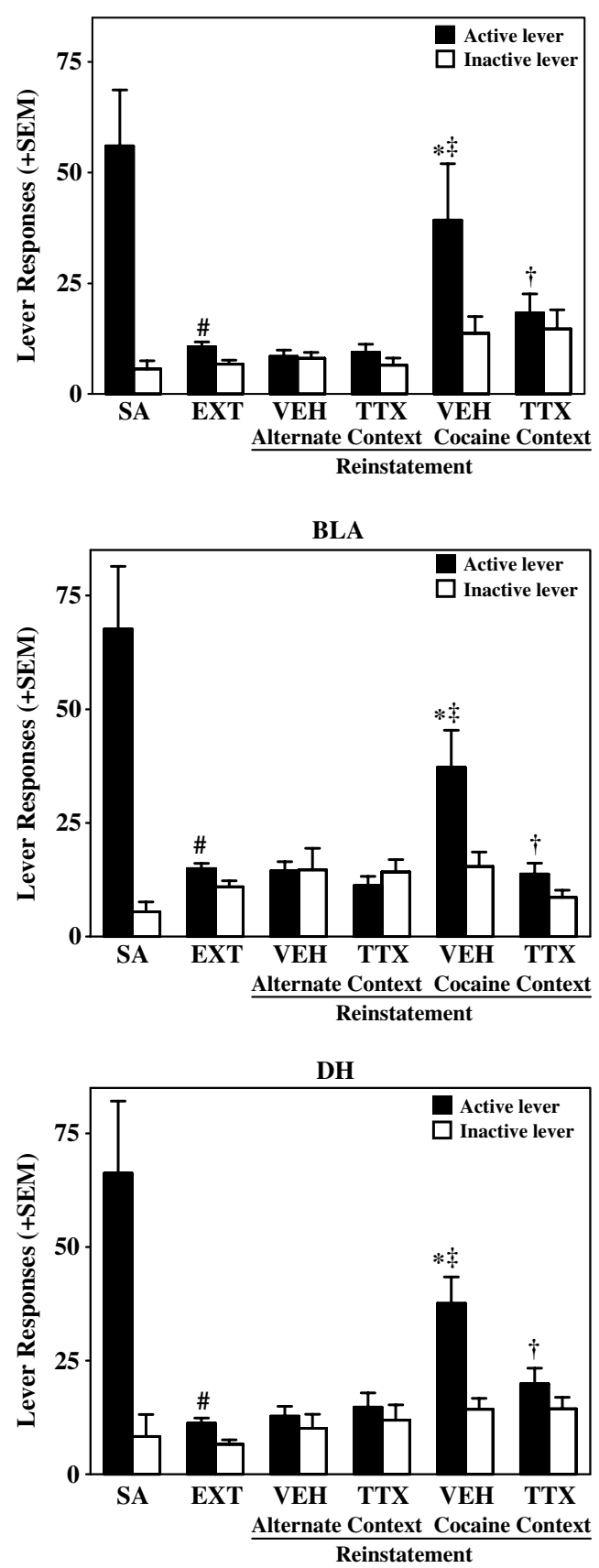
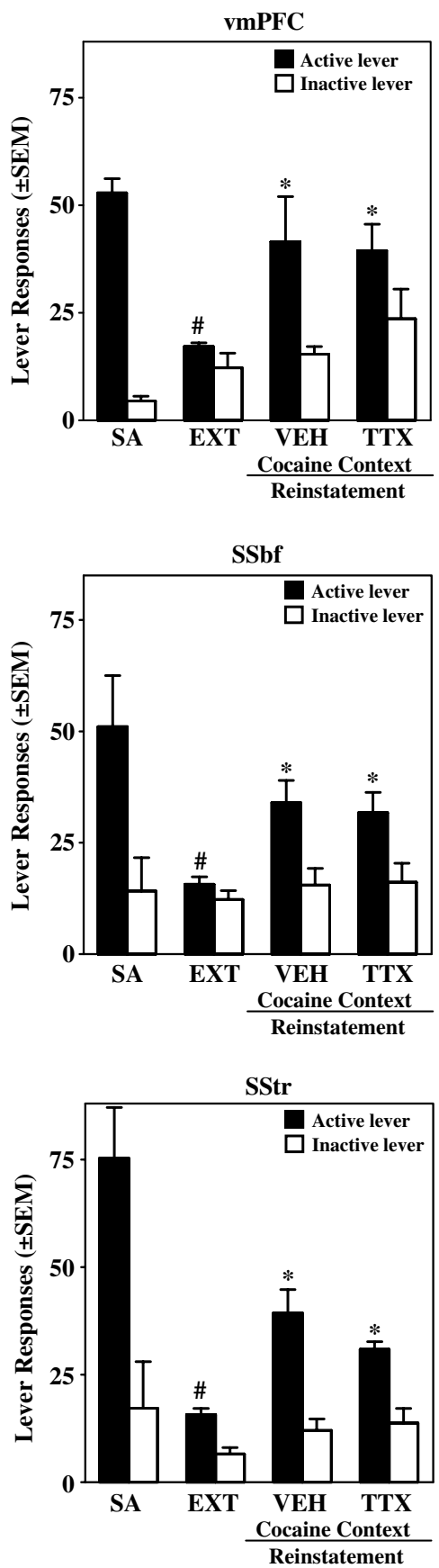

Figure 3 Responses on the active and inactive levers ( \pm SEM) during self-administration (SA; average of the last 3 days), extinction (EXT; average of the last extinction day preceding the TTX reinstatement test day and the last extinction day preceding the vehicle reinstatement test day), and contextual reinstatement testing. On the test day, rats received intracranial pretreatment with TTX or vehicle and were then exposed to the previously cocaine-paired context ( $N=7-10 /$ brain region) or the alternate context $(N=8-10 /$ brain region) in the absence of cocaine reinforcement or response-contingent stimulus presentation. Symbols represent significant difference relative to responding during self-administration (\#, ANOVA day simple main effects test, $P<0.05$ ), during extinction (*, Tukey test, $P<0.05$; SSbf and vmPFC, $t$-test, $P<0.05$ ), upon exposure to the alternate context ( $\ddagger$, Tukey test, $P<0.05$ ), and relative to vehicle pretreatment $(\dagger$, Tukey test, $P<0.05)$.

a significant day by lever interaction effect $\left(\mathrm{F}_{(2,14)}=9.11\right.$, $P<0.005)$, and significant main effects of day $\left(\mathrm{F}_{(2,14)}=\right.$ 11.09, $P<0.001)$ and lever $\left(\mathrm{F}_{(1,7)}=22.36, \quad P<0.005\right)$. Subsequent pairwise comparisons revealed that responsecontingent presentation of the light-tone CSs following vehicle or TTX infusions into the DH produced a significant increase in responding on the active lever relative to responding during extinction (Tukey test, $P<0.05$ ) and relative to the inactive lever (Tukey test, $P<0.05$ ). Thus, TTX pretreatment infused into the $\mathrm{DH}$ failed to alter the reinstatement in responding on the active lever elicited by the light-tone CSs relative to vehicle (Tukey test, $P>0.5$ ). Furthermore, TTX pretreatment infused into the DH failed to alter responding on the active lever during the 


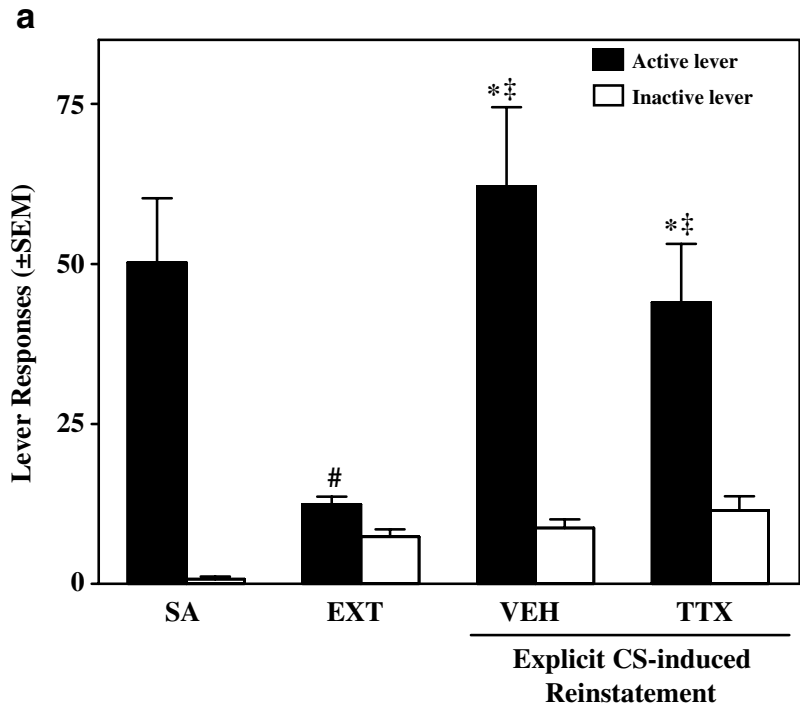

b

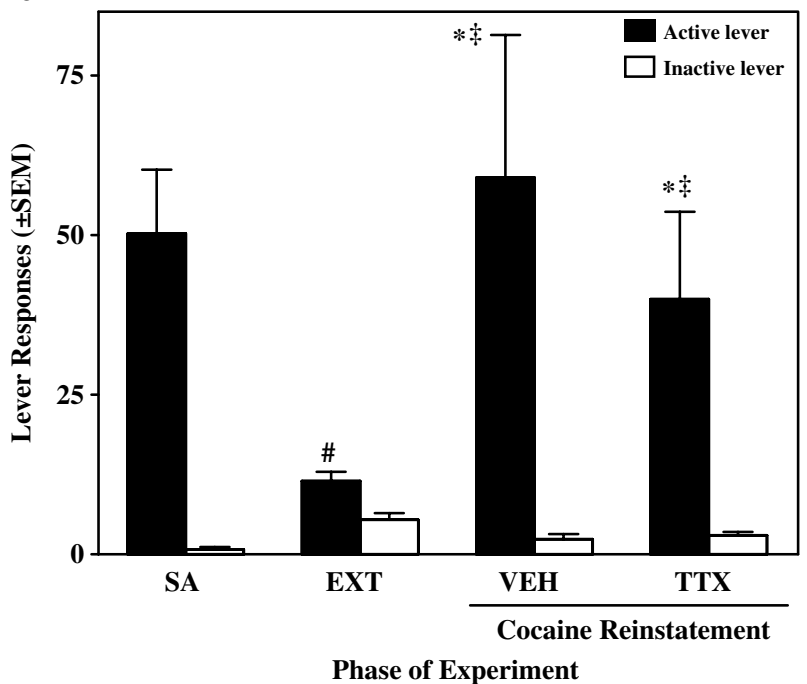

Figure 4 Responses on the active and inactive levers ( \pm SEM) during self-administration (SA; average of the last 3 days), extinction (EXT; average of the last extinction day preceding the TTX reinstatement test day and the last extinction day preceding the vehicle reinstatement test day), and reinstatement testing. On the explicit CS-induced reinstatement test day (a), rats $(N=8)$ received an infusion of TTX or vehicle into the $\mathrm{DH}$, and responses on the active lever resulted in presentations of the light-tone stimulus complex in the absence of cocaine reinforcement. On the cocaine reinstatement test day $(b)$, rats $(N=8)$ received an infusion $T T X$ or vehicle into the $\mathrm{DH}$ and a systemic infusion of cocaine $(10 \mathrm{mg} / \mathrm{kg}$, i.p.); responses had no programmed consequences. Symbols represent significant difference relative to responding during self-administration (\#, ANOVA day simple main effects test, $P<0.05$ ), during extinction (*a: Tukey test, $P<0.05$; b: planned t-test, $P<0.5)$, and relative to the inactive lever $(\ddagger$, a: Tukey test, $P<0.05$; b: planned $t$-test, $P<0.5$ ).

reinstatement test relative to responding during selfadministration $\left(t_{(7)}=0.37, P>0.05\right)$. Pairwise comparisons of responses on the inactive lever did not reveal a day effect. The effects of intra-DH infusions of TTX were not examined on extinction responding in the absence of cue presentation. However, given that inactivation of the $\mathrm{DH}$ failed to alter locomotion (see below) and operant responding on the inactive lever (see above), it is unlikely that the observed lack of an effect on active lever responding was due to nonspecific effects on general activity.

Cocaine-primed reinstatement of extinguished cocaineseeking behavior. TTX inactivation of the DH failed to alter the ability of the cocaine priming injection to reinstate responding (Figure $4 \mathrm{~b}$ ). Cocaine-primed reinstatement of responding was not due to the stress of intraperitoneal drug administration since intraperitoneal administration of saline alone or in combination with intracranial administration of saline fails to reinstate responding (Neisewander et al, 2000; Fuchs et al, 2002a; Fuchs and See, 2002; Ledford et al, 2003).

There was no significant difference in responding during the extinction days that immediately preceded the vehicle and TTX reinstatement test days; therefore, extinction data were collapsed across these time points (extinction, henceforth). The $3 \times 2$ repeated measures ANOVA of lever presses during the reinstatement test days and extinction indicated a significant main effect of lever $\left(\mathrm{F}_{(1,14)}=17.326\right.$, $P<0.005)$ and a trend for a day by lever interaction effect $\left(\mathrm{F}_{(2,14)}=2.64, \quad P=0.10\right)$, but no day main effect $\left(\mathrm{F}_{(2,14)}=1.89, P=0.19\right)$. Subsequent planned comparisons indicated that cocaine priming following vehicle or TTX infusions into the $\mathrm{DH}$ produced a significant increase in responding on the active lever relative to responding during extinction (Vehicle, one-tail $t_{(7)}=2.07, P<0.05$; TTX, onetail $\left.t_{(7)}=2.13, P<0.05\right)$ and relative to the inactive lever (Vehicle, $t_{(7)}=2.53, P<0.05$; TTX, $t_{(7)}=2.51, P<0.05$ ). Thus, TTX pretreatment infused into the DH failed to alter the reinstatement in responding on the active lever elicited by cocaine priming relative to vehicle $\left(t_{(7)}=0.67, P>0.05\right)$. Furthermore, TTX pretreatment infused into the DH failed to alter responding on the active lever during the reinstatement test relative to responding during selfadministration $\left(t_{(7)}=0.59, P>0.05\right)$. Given that inactivation of the DH failed to alter locomotion (see below) and operant responding on the inactive lever (see above), it is unlikely that the observed lack of an effect on active lever responding was due to nonspecific effects on general activity.

Locomotor activity. TTX infused into the dmPFC, DH, vmPFC, SStr, or SSbf did not alter locomotor activity (Figure 5), similar to findings obtained following infusion of TTX into the BLA (McLaughlin and See, 2003). Separate $2 \times 6$ mixed factor ANOVAs of photobeam breaks indicated significant main effects of time $\left(\mathrm{dmPFC}, \mathrm{F}_{(5,40)}=39.89\right.$, $P<0.0001 ; \quad \mathrm{DH}, \quad \mathrm{F}_{(5,45)}=43.58, \quad P<0.0001 ; \quad$ vmPFC, $\mathrm{F}_{(5,50)}=36.02, P<0.0001 ;$ SStr, $\mathrm{F}_{(5,45)}=36.47, P<0.0001$; SSbf, $\left.\mathrm{F}_{(5,50)}=21.78, P<0.0001\right)$, but no significant main effects of group or time by group interaction effects. Thus, locomotor activity gradually decreased across the 1-h test. TTX pretreatment infused into the dmPFC, DH, vmPFC, SSbf, or SStr failed to significantly alter locomotor activity relative to vehicle pretreatment, although TTX pretreatment infused into the SStr produced a nonsignificant trend toward a decrease in locomotion (ANOVA treatment main effect, $\left.\mathrm{F}_{(1,9)}=3.69, P=0.08\right)$. 

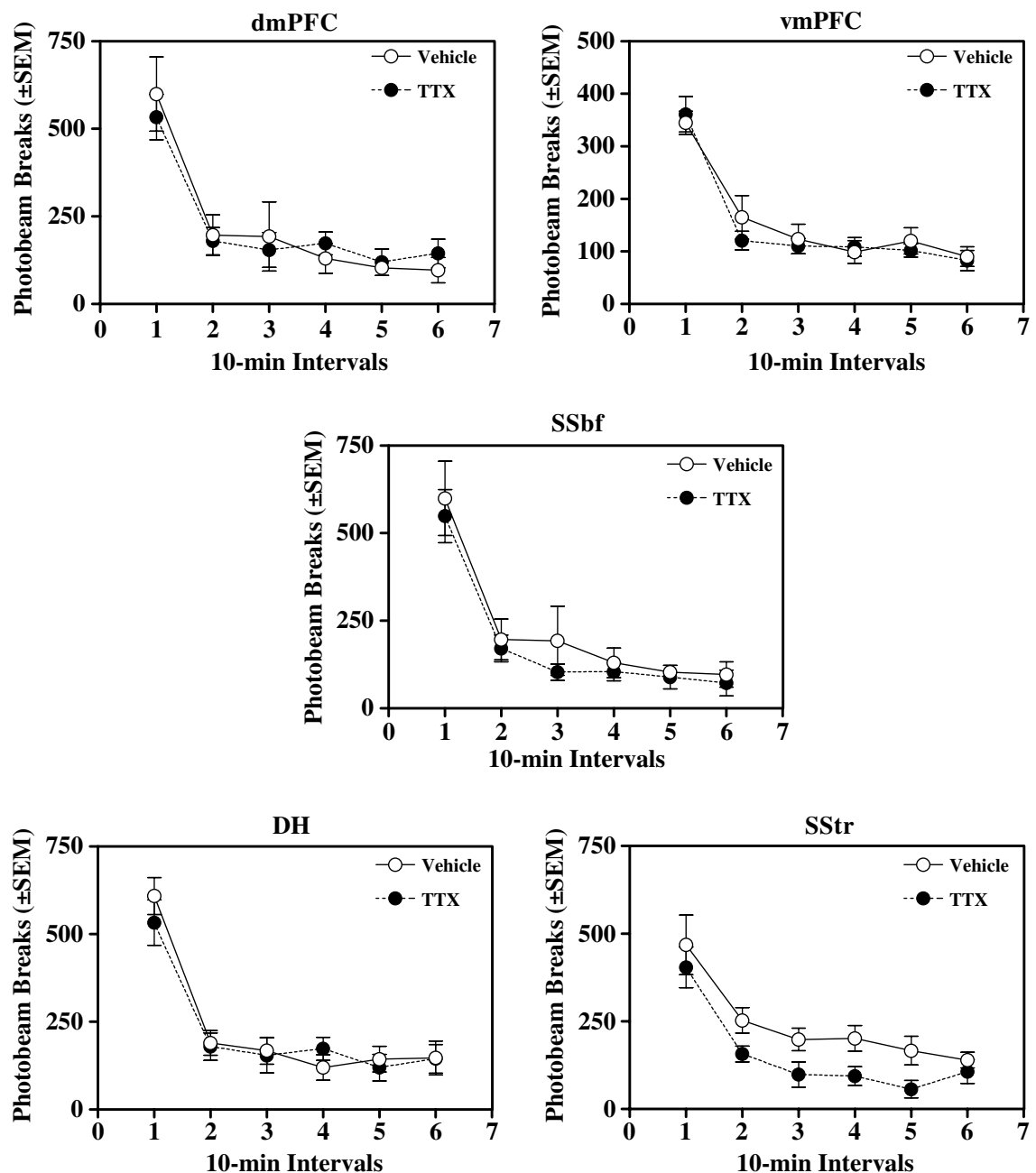

Figure 5 Locomotor activity during a I-h test session following intracranial pretreatment with $T T X(N=5-6 / b r a i n ~ r e g i o n)$ or vehicle $(N=5-6 / b r a i n$ region). An automated photocell system recorded the number of photobeam interruptions elicited by a rat moving in the chamber. TTX infused into the dmPFC, DH, vmPFC, SStr, or SSbf failed to alter this measure of locomotor activity, similar to findings obtained following infusion of TTX into the BLA (McLaughlin and See, 2003).

\section{DISCUSSION}

\section{Contextual Reinstatement of Cocaine-Seeking Behavior}

The present study is first to map the neural circuitry of contextual reinstatement of cocaine-seeking behavior and to compare this circuitry to the neural substrates of explicit CS- and cocaine-induced cocaine-seeking behavior. Rats were initially trained to self-administer cocaine in a distinct environmental context, then underwent extinction training in the previously cocaine-paired context or in an alternate context. Consistent with previous research (Crombag et al, 2002), the magnitudes of extinction responding and the rates of extinction exhibited by the groups that underwent extinction training in the previously cocaine-paired $v s$ alternate contexts were similar. However, presentation of the cocaine-paired contextual stimuli selectively reinstated extinguished cocaine-seeking behavior. Reinstatement was not due to dishabituation precipitated by a change in testing environment, since presentation of the alternate context failed to alter responding. TTX-induced inactivation of the
dmPFC, BLA, or DH inhibited contextual reinstatement of cocaine seeking, while inactivation of the $\mathrm{DH}$ failed to alter explicit CS-induced and cocaine-primed reinstatement.

Before comparing the neural substrates of cocaineseeking behavior elicited by contexts $v s$ explicit CSs based on the above observations, we must consider that cocaineassociated contexts $v s$ explicit CSs varied in several respects in the present study as well as in the natural environment of human drug users. First, these manipulations differed in sensory demand and complexity. A more appropriate comparison between contextual $S_{D} S$ and CSs of identical sensory demand and complexity could not be made because our preliminary studies indicated that a context consisting of only auditory and visual stimuli was insufficient to significantly reinstate cocaine-seeking behavior in control rats unlike the light-tone CSs presented response contingently. Therefore, the complexity of the context was increased in an attempt to facilitate context-reward learning by increasing stimulus salience (Rescorla and Wagner, 1972). Conversely, the olfactory and tactile stimuli could not be included as components of a multimodal explicit CS 
complex because of technical difficulties associated with their response-contingent presentation. Second, contexts and explicit CSs were administered using different modes of stimulus presentation. Contexts were presented independent of responding, whereas explicit CSs had to be presented response-contingently because passive presentation of these stimuli results in little or no cocaine-seeking behavior (Tran-Nguyen et al, 1998; Grimm et al, 2000). These differences in stimulus presentation are important because they likely determine the relationship of these stimuli to the US. Cocaine-paired stimuli presented response-contingently can act as conditioned reinforcers (ie, stimuli that elicit responding with the goal of obtaining further cue presentations) or conditioned incentive motivational stimuli (ie, stimuli that elicit responding with the goal of obtaining the US) (Mackintosh, 1974; Markou et al, 1993). In contrast, contexts engender conditioned behaviors primarily by acting as occasion setters, or $\mathrm{S}_{\mathrm{D}} \mathrm{s}$ that retrieve response-US and/or explicit CS-US associations learned in those contexts (Bouton and Swartzentruber, 1986; Gordon and Klein, 1994; Robinson and Berridge, 2003). Thus, they signal drug availability contingent upon responding and likely act as conditioned incentive motivational stimuli as opposed to conditioned reinforcers. Based on these differences, it has been hypothesized that $S_{D} s$, such as contexts, initiate relapse to drug seeking whereas explicit CSs are critical to the maintenance of drug seeking (Di Ciano and Everitt, 2003).

Emerging neurochemical and behavioral findings support the idea that $S_{D} S$ and explicit CSs act through separate mechanisms (See et al, 1999; Ito et al, 2000; Di Ciano and Everitt, 2003; Bossert et al, 2004). In contrast to previous studies utilizing the renewal model and discriminative stimulus training procedures, in which explicit CS-US pairings occurred in the presence of contextual or discrete $\mathrm{S}_{\mathrm{D}}$ s (Weiss et al, 2000; Ciccocioppo et al, 2001; Crombag et al, 2002; Crombag and Shaham, 2002; Yun and Fields, 2003), explicit CSs in the present study were not programmed to occur in the drug-paired context allowing isolation of the motivational effects of the context from those of explicit CSs. Under these conditions, strong context-response-US associations formed in the drugpaired context, enabling the context to reinstate cocaineseeking behavior. The finding that $\mathrm{DH}$ inactivation selectively abolished contextual reinstatement of extinguished cocaine-seeking behavior is consistent with the argument that context acted as an occasion setter or 'background stimulus' as opposed to a CS paired directly with cocaine (see below).

\section{Contributions of the DH, BLA, and dmPFC to Cocaine-Seeking Behavior}

Functional inactivation of the dmPFC or BLA abolished the ability of the cocaine-paired context to elicit cocaineseeking behavior (Figure 3), similar to the effects of dmPFC or BLA inactivation on explicit CS-induced reinstatement of cocaine-seeking behavior (McLaughlin and See, 2003). In contrast, inactivation of the $\mathrm{DH}$ abolished contextual reinstatement (Figure 3), but failed to alter explicit CS- or cocaine-induced reinstatement, of cocaine-seeking behavior (Figure 4). It is unlikely that the TTX-induced impairments in cocaine-seeking behavior were due to nonspecific performance deficits, since TTX impaired operant responding in a lever- and stimulus-specific manner and failed to alter locomotor activity (Figure 5, McLaughlin and See, 2003). Furthermore, the effects of TTX were anatomically specific, since TTX failed to impair responding when administered to brain regions adjacent and/or dorsal to the target brain regions (ie, vmPFC, SSbf, SStr). Overall, the findings support the hypothesis that unique but partially overlapping brain systems mediate the motivational effects of drug contexts $v s$ explicit CSs on cocaine-seeking behavior. Furthermore, the DH, BLA, and dmPFC appear to contribute to cocaine-seeking behavior with varying degree of specificity regarding the type of manipulation that is used to trigger relapse (see below).

The present study suggests that the functional integrity of the DH is necessary for expression of context-induced, but not explicit CS- or cocaine-induced, cocaine-seeking behavior. The differential effects of $\mathrm{DH}$ inactivation on contextual vs explicit CS-induced reinstatement were not likely due to differences in stimulus modality, since the $\mathrm{DH}$ receives rich multimodal sensory input from sensory cortical association areas (Moser and Moser, 1998). Instead, these effects likely reflect the differential involvement of the $\mathrm{DH}$ in various forms of associative learning. The $\mathrm{DH}$ is not necessary for explicit CS-US or 'foreground' contextual conditioning since post-training DH lesions or inactivation fail to impair conditioned fear both to explicit (Kim and Fanselow, 1992; Maren et al, 1997) and contextual CSs paired directly with shock (Phillips and LeDoux, 1994), and fail to impair contextual discrimination performance (Good and Honey, 1991). In contrast, the integrity of the $\mathrm{DH}$ is necessary for 'background' contextual fear conditioning, since post-training DH lesions abolish conditioned fear to a context that previously served as a background to CS-shock pairings and impair context-dependent latent inhibition to an explicit CS (Kim and Fanselow, 1992; Phillips and LeDoux, 1994; Maren et al, 1997; Holt and Maren, 1999). The DH interacts with subcortical brain regions via its output structure, the dorsal subiculum (Jay et al, 1992; Ferbinteanu and McDonald, 2001). Thus, it is somewhat surprising that dorsal subiculum inactivation fails to alter cocaine-seeking behavior elicited by a combination of a light-tone contextual $S_{D}$ and an explicit $C S$, or by a combination of these stimuli and a cocaine priming injection (Black et al, 2004). Cocaine seeking is expected to be maintained, in part, by the $S_{D} s$ under these testing conditions; thus, these findings appear to be inconsistent with those of Experiment 1. However, it is possible that a bimodal $S_{D}$ does not exert significant control over cocaine seeking in the presence of a more salient explicit CS or a cocaine priming injection and, under these conditions, cocaine-seeking behavior does not depend on the functional integrity of the $\mathrm{DH}$ as shown in Experiment 2. Alternatively, cocaine-seeking behavior is perhaps controlled by the $S_{D}$, but the $\mathrm{DH}$ mediates contextual reinstatement via its cortically projecting output region, the lateral entorhinal cortex, as opposed to the dorsal subiculum (Jay et al, 1992; Ferbinteanu and McDonald, 2001). Overall, these and the present findings suggest that the $\mathrm{DH}$ does not appear to mediate the incentive motivational effects of explicit cocaine-paired CSs or cocaine itself. However, consistent 
with its theorized role in spatial and configular associations (Sutherland et al, 1989; Sutherland and McDonald, 1990; Rolls, 2000), the DH mediates expression of cocaine-seeking behavior elicited by contextual background stimuli and/or occasion setters. Future studies will need to elucidate the involvement of other regions of the hippocampal formation in context-induced cocaine seeking.

Previous studies suggest that the functional integrity of the BLA is necessary for the maintenance of stimulusreward associations that underlies the ability of cocainepaired stimuli to elicit goal-directed behaviors. Consistent with this characterization, rostral BLA lesions or inactivation also abolish expression of cocaine-seeking behavior elicited by discrete cocaine-predictive $\mathrm{S}_{\mathrm{D}} \mathrm{s}$ (Kantak et al, 2002) or explicit CSs (Meil and See, 1997; Grimm and See, 2000; McLaughlin and See, 2003) and spatial place conditioning (White and McDonald, 1993). Similarly, post-training BLA lesions abolish expression of conditioned fear to a shock-paired context as well as to an explicit CS (Maren, 1998, 2001), suggesting that the integrity of the rostral BLA is necessary for the expression of previously established context-US as well as discrete CS-US associations. However, caudal BLA lesions or inactivation fail to abolish cocaine-seeking behavior elicited by cocaine-predictive stimuli (Fuchs et al, 2002b; Kantak et al, 2002) and impair extinction of cocaine place conditioning (Fuchs et al, 2002b; but see Hiroi and White, 1991). In the present study, BLA inactivation abolished expression of contextual reinstatement of extinguished cocaine-seeking, similar to the effects of rostral BLA inactivation on explicit CS-induced reinstatement. Alternatively, BLA inactivation may have abolished contextual reinstatement by impairing the rat's ability to solve this task by treating components of the context as a constellation of CSs. However, the idea that rats simply processed the context as a collection of CSs is inconsistent with the finding that $\mathrm{DH}$ inactivation had a differential effect on contextual $v s$ explicit CS-induced reinstatement of cocaine seeking. Thus, collectively, the above findings suggest that the contribution of the BLA to cocaine-seeking behavior is subregion specific, and less specialized than that of the $\mathrm{DH}$. Unlike the $\mathrm{DH}$, portions of the BLA are involved in determining the incentive motivational significance of both cocaine-paired contextual and explicit CSs.

Inactivation of the $\mathrm{dmPFC}$, which targeted the anterior cingulate and dorsal prelimbic cortices, abolished contextual reinstatement of cocaine-seeking behavior. This finding adds to a growing literature indicating that the functional integrity of the dmPFC is necessary for cocaine-seeking behavior. For instance, impairment of dmPFC function disrupts stimulus control over operant responding on second-order schedules of cocaine reinforcement (Weissenborn et al, 1997) and attenuates explicit CS-induced reinstatement of cocaine-seeking behavior (McLaughlin and See, 2003). Additionally, the dmPFC exhibits neural activation in response to both cocaine-paired stimuli and cocaine priming injections (Brown et al, 1992; Neisewander et al, 2000; Ciccocioppo et al, 2001) and the functional integrity of this structure is necessary for cocaine-primed reinstatement of cocaine-seeking behavior (McFarland and Kalivas, 2001), unlike the BLA or DH (Figure 3b; Grimm and See, 2000; Neisewander et al, 2000; McFarland and
Kalivas, 2001, but see Kantak et al, 2002; Yun and Fields, 2003). Thus, among the three structures examined, the dmPFC is the most generalized in its involvement in cocaine-seeking behavior which is consistent with its theorized role in executive functions, including attentional processes and response selection (Janer and Pardo, 1991; Devinsky et al, 1995; Bolla et al, 1998). Specifically, the dmPFC is thought to modulate the salience and motivational significance of stimuli by regulating attention to the sensory input that enters the BLA and hippocampus (Kolb, 1984; Rosenkranz and Grace, 2001) and by mediating BLA input to the nucleus accumbens (Jackson and Moghaddam, 2001), respectively. Furthermore, it has been theorized that the dmPFC initiates cocaine-seeking behavior in response to motivational stimuli, including cocaine-paired contextual $\mathrm{S}_{\mathrm{D}} \mathrm{S}$, explicit CSs, or cocaine, via its extensive projections to the spinal cord and the basal ganglia (Devinsky et al, 1995; Bolla et al, 1998), which may mediate the compulsive, automated aspect of cocaine-seeking behavior (McFarland and Kalivas, 2001).

Contextual reinstatement of cocaine-seeking behavior likely involves interaction of the DH, BLA, dmPFC, as well as sensory and motor structures, because following the inactivation of any of these three structures, functional integrity of the remaining two brain regions was insufficient to maintain context-induced reinstatement. However, a series of functional disconnection experiments will need to be conducted in order to determine the exact functional connectivity between these brain regions. Nevertheless, the main finding of the present study is that distinct, but partially overlapping, neural substrates mediate the motivational effects of cocaine-paired contexts, cocaine-paired explicit CSs, and cocaine; therefore, the development of pharmacotherapies that selectively target the neural substrates of context-induced relapse may be possible. Future research will need to elucidate the neuropharmacological mechanism of contextual reinstatement within the $\mathrm{DH}$, BLA, and dmPFC in order to provide further information for treatment development for drug dependence.

\section{ACKNOWLEDGEMENTS}

We would like to thank William Berglind for excellent technical assistance. This work was supported by National Institute on Drug Abuse Grant DA10462.

\section{REFERENCES}

Alleweireldt AT, Weber SM, Neisewander JL (2001). Passive exposure to a contextual discriminative stimulus reinstates cocaine-seeking behavior in rats. Pharmacol Biochem Behav 69: 555-560.

Ambrogi Lorenzini CG, Baldi E, Bucherelli C, Tassoni G (1995). Time-dependent deficits of rat's memory consolidation induced by tetrodotoxin injections into the caudate-putamen, nucleus accumbens and globus pallidus. Neurobiol Learn Mem 63: 87-93.

Black YD, Green-Jordan K, Eichenbaum HB, Kantak KM (2004). Hippocampal memory system function and the regulation of cocaine self-administration behavior in rats. Behav Brain Res 151: 225-238. 
Bolla KI, Cadet J-L, London ED (1998). The neuropsychiatry of chronic cocaine abuse. J Neuropsych Clin Neurosci 10: 280-289.

Bossert JM, Liu SY, Shaham Y (2004). The mGluR2/3 agonist, Ly379268, attenuates reinstatement of heroin and sucrose seeking induced by re-exposure to drug-associated contextual cues in rats. Submitted.

Bouton ME, Bolles RC (1979). Role of conditioned contextual stimuli in reinstatement of extinguished fear. J Exp Psychol 5: 368-378.

Bouton ME, Ricker ST (1994). Renewal of extinguished responding in a second context. Anim Learn Behav 22: 317-324.

Bouton ME, Swartzentruber D (1986). Analysis of the associative and occasion-setting properties of contexts participating in a Pavlovian discrimination. J Exp Psychol 12: 333-350.

Brown EE, Robertson GS, Fibiger HC (1992). Evidence for conditional neuronal activation following exposure to a cocaine-paired environment: role of forebrain limbic structures. J Neurosci 12: 4112-4121.

Cahill L, Coopersmith RM, Leon M, McGaugh JL (1987). Local injection of tetrodotoxin decreases metabolic activity in discrete brain regions: A 2-deoxyglucose autoradiography analysis. Soc Neurosci Abst 13: 1414.

Ciccocioppo R, Sanna PP, Weiss F (2001). Cocaine-predictive stimulus induces drug-seeking behavior and neural activation in limbic brain regions after multiple months of abstinence: reversal by D1 antagonists. Proc Natl Acad Sci USA 98: 1976-1981.

Cornish JL, Duffy P, Kalivas PW (1999). A role for nucleus accumbens glutamate transmission in the relapse to cocaineseeking behavior. Neuroscience 93: 1359-1367.

Crombag HS, Grimm JW, Shaham Y (2002). Effect of dopamine receptor antagonists on renewal of cocaine seeking by reexposure to drug-associated contextual cues. Neuropsychopharmacology 27: 1006-1015.

Crombag HS, Shaham Y (2002). Renewal of drug seeking by contextual cues after prolonged extinction in rats. Behav Neurosci 116: 169-173.

de Wit H, Stewart J (1981). Reinstatement of cocaine-reinforced responding in the rat. Psychopharmacology 75: 134-143.

Devinsky O, Morrell MJ, Vogt BA (1995). Contributions of anterior cingulate cortex to behaviour. Brain 118: 279-306.

Di Ciano P, Everitt BJ (2003). Differential control over drugseeking behavior by drug-associated conditioned reinforcers and discriminative stimuli predictive of drug availability. Behav Neurosci 117: 952-960.

Ehrman RN, Robbins SJ, Childress AR, O’Brien CP (1992). Conditioned responses to cocaine-related stimuli in cocaine abuse patients. Psychopharmacology 107: 523-529.

Ferbinteanu J, McDonald RJ (2001). Dorsal/ventral hippocampus, fornix, and conditioned place preference. Hippocampus 11: 187-200.

Foltin RW, Haney M (2000). Conditioned effects of environmental stimuli paired with smoked cocaine in humans. Psychopharmacology 149: 24-33.

Fuchs R, Tran-Nguyen L, Weber S, Khroyan T, Neisewander J (2002a). Effects of 7-OH-DPAT on cocaine-seeking behavior and on re-establishment of cocaine self-administration. Pharmacol Biochem Behav 72: 623-632.

Fuchs RA, See RE (2002). Basolateral amygdala inactivation abolishes conditioned stimulus- and heroin-induced reinstatement of extinguished heroin-seeking behavior in rats. Psychopharmacology 160: 425-433.

Fuchs RA, Weber SM, Rice HJ, Neisewander JL (2002b). Effects of excitotoxic lesions of the basolateral amygdala on cocaineseeking behavior and cocaine conditioned place preference in rats. Brain Res 929: 15-25.

Gerber GJ, Stretch R (1975). Drug-induced reinstatement of extinguished self-administration behavior in monkeys. Pharmacol Biochem Behav 3: 1055-1061.
Goddard MJ (1999). Renewal to the signal value of an unconditioned stimulus. Learn Motiv 30: 15-34.

Good M, Honey RG (1991). Conditioning and contextual retrieval in hippocampal rats. Behav Neurosci 105: 499-509.

Gordon WC, Klein RL eds (1994). Animal Memory. The Effects of Context Change on Retention Performance, 2 edn. Academic Press: San Diego.

Grimm JW, Kruzich PJ, See RE (2000). Contingent access to stimuli associated with cocaine self-administration is required for reinstatement of drug-seeking behavior. Psychobiology 28: 383-386.

Grimm JW, See RE (2000). Dissociation of primary and secondary reward-relevant limbic nuclei in an animal model of relapse. Neuropsychopharmacology 22: 473-479.

Hiroi N, White NM (1991). The lateral nucleus of the amygdala mediates expression of the amphetamine-produced conditioned place preference. J Neurosci 11: 2107-2116.

Holt W, Maren S (1999). Muscimol inactivation of the dorsal hippocampus impairs contextual retrieval of fear memory. $J$ Neurosci 19: 9054-9062.

Ito R, Dalley JW, Howes SR, Robbins TW, Everitt BJ (2000). Dissociation in conditioned dopamine release in the nucleus accumbens core and shell in response to cocaine cues and during cocaine-seeking behavior in rats. J Neurosci 20: 7489-7495.

Jackson ME, Moghaddam B (2001). Amygdala regulation of nucleus accumbens dopamine output is governed by the prefrontal cortex. J Neurosci 21: 676-681.

Jaffe JH, Cascella NG, Kumor KM, Sherer MA (1989). Cocaineinduced cocaine craving. Psychopharmacology 97: 59-64.

Janer KW, Pardo JV (1991). Deficits in selective attention following bilateral anterior cingulotomy. J Cogn Neurosci 3: 231-241.

Jay TM, Thierry AM, Wiklund L, Glowinski J (1992). Excitatory amino acid pathway from the hippocampus to the prefrontal cortex. Contribution of AMPA receptors in hippocampoprefrontal cortex transmission. Eur J Neurosci 4: 1285-1295.

Kantak KM, Black Y, Valencia E, Green-Jordan K, Eichenbaum HB (2002). Dissociable effects of lidocaine inactivation of the rostral and caudal basolateral amygdala on the maintenance and reinstatement of cocaine-seeking behavior in rats. $J$ Neurosci 22: 1126-1136.

Kim JJ, Fanselow MS (1992). Modality-specific retrograde amnesia of fear. Science 256: 675-677.

Kolb B (1984). Functions of the frontal cortex of the rat: a comparative review. Brain Res Rev 8: 65-98.

Kruzich PJ, See RE (2001). Differential contributions of the basolateral and central amygdala in the acquisition and expression of conditioned relapse to cocaine-seeking behavior. J Neurosci 21RC155: 1-5.

Ledford CC, Fuchs RA, See RE (2003). Potentiated reinstatement of cocaine-seeking behavior following $\mathrm{D}$-amphetamine infusion into the basolateral amygdala. Neuropsychopharmacology 28: 1721-1729.

Mackintosh NJ (1974). The Psychology of Animal Learning. Academic Press: New York.

Maren S (1998). Overtraining does not mitigate contextual fear conditioning deficits produced by neurotoxic lesions of the basolateral amygdala. J Neurosci 18: 3088-3097.

Maren S (2001). Is there savings for Pavlovian fear conditioning after neurotoxic basolateral amygdala lesions in rats? Neurobiol Learn Mem 76: 268-283.

Maren S, Aharonov G, Fanselow MS (1997). Neurotoxic lesions of the dorsal hippocampus and Pavlovian fear conditioning in rats. Behav Brain Res 88: 261-274.

Markou A, Weiss F, Gold LH, Caine SB, Schulteis G, Koob GF (1993). Animal models of drug craving. Psychopharmacology 112: $163-182$.

Martin JH, Ghez C (1999). Pharmacological inactivation in the analysis of the central control of movement. J Neurosci Methods 86: 145-159. 
McFarland K, Kalivas PW (2001). The circuitry mediating cocaineinduced reinstatement of drug-seeking behavior. J Neurosci 21: 8655-8663.

McLaughlin J, See RE (2003). Selective inactivation of the dorsomedial prefrontal cortex and the basolateral amygdala attenuates conditioned-cued reinstatement of extinguished cocaine-seeking behavior in rats. Psychopharmacology 168: 57-65.

Meil WM, See RE (1997). Lesions of the basolateral amygdala abolish the ability of drug associated cues to reinstate responding during withdrawal from self-administered cocaine. Behav Brain Res 87: 139-148.

Meyers RA, Zavala AR, Neisewander JL (2003). Dorsal, but not ventral, hippocampal lesions disrupt cocaine place conditioning. NeuroReport 14: 2127-2131.

Moser MB, Moser EI (1998). Functional differentiation in the hippocampus. Hippocampus 8: 608-619.

Nakajima S, Tanaka S, Urushihara K, Imada H (2000). Renewal of extinguished lever-press responses upon return to the training context. Learn Motiv 31: 416-431.

Neisewander JL, Baker DA, Fuchs RA, Tran-Nguyen LT, Palmer A, Marshall JF (2000). Fos protein expression and cocaine-seeking behavior in rats after exposure to a cocaine self-administration environment. J Neurosci 20: 798-805.

Paxinos G, Watson C (1997). The Rat Brain in Stereotaxic Coordinates. Academic Press: San Diego, CA.

Phillips RG, LeDoux JE (1994). Lesions of the dorsal hippocampal formation interfere with background but not foreground contextual fear conditioning. Learn Mem 1: 34-44.

Rauhut AS, Castle B, Fenton L, Bardo MT (2000). Renewal: a novel animal model of drug relapse. Soc Neuro Abstr 482: 481.

Rescorla RA, Wagner AR (eds) (1972). A Theory of Pavlovian Conditioning: Variations in the Effectiveness of Reinforcement and Nonreinforcement. Appleton-Century-Crofts: New York.

Robinson TE, Berridge KC (2003). Addiction. Annu Rev Psychol 54: 25-53.

Rolls ET (2000). Memory systems in the brain. Annu Rev Psychol 51: 599-630.

Rosenkranz JA, Grace AA (2001). Dopamine attenuates prefrontal cortical suppression of sensory inputs to the basolateral amygdala in rats. J Neurosci 21: 4090-4103.

See RE, Grimm JW, Kruzich PJ, Rustay N (1999). The importance of a compound stimulus in conditioned drug-seeking behavior following one week of extinction from self-administered cocaine in rats. Drug Alcohol Depend 57: 41-49.
See RE, Kruzich PJ, Grimm JW (2001). Dopamine, but not glutamate, receptor blockade in the basolateral amygdala attenuates conditioned reward in a rat model of relapse to cocaine-seeking behavior. Psychopharmacology 154: 301-310.

Shalev U, Grimm JW, Shaham Y (2002). Neurobiology of relapse to heroin and cocaine seeking: a review. Pharmacol Rev 54: 1-42.

Sutherland RJ, McDonald RJ (1990). Hippocampus, amygdala, and memory deficits in rats. Behav Brain Res 37: 57-79.

Sutherland RJ, McDonald RJ, Hill CR, Rudy JW (1989). Damage to the hippocampal formation in rats selectively impairs the ability to learn cue relationships. Behav Neural Biol 52: 331-356.

Tran-Nguyen LT, Fuchs RA, Coffey GP, Baker DA, O'Dell LE, Neisewander JL (1998). Time-dependent changes in cocaineseeking behavior and extracellular dopamine levels in the amygdala during cocaine withdrawal. Neuropsychopharmacology 19: $48-59$.

Tzschentke TM, Schmidt WJ (1999). Functional heterogeneity of the rat medial prefrontal cortex: effects of discrete subarea-specific lesions on drug-induced conditioned place preference and behavioural sensitization. Eur J Neurosci 11: 4099-4109.

Weiss F, Maldonado-Vlaar CS, Parsons LH, Kerr TM, Smith DL, Ben-Shahar O (2000). Control of cocaine-seeking behavior by drug-associated stimuli in rats: effects on recovery of extinguished operant-responding and extracellular dopamine levels in amygdala and nucleus accumbens. Proc Natl Acad Sci USA 97: 4321-4326.

Weissenborn R, Robbins TW, Everitt BJ (1997). Effects of medial prefrontal or anterior cingulate cortex lesions on responding for cocaine under fixed-ratio and second-order schedules of reinforcement in rats. Psychopharmacology 134: 242-257.

White NM, McDonald RJ (1993). Acquisition of a spatial conditioned place preference is impaired by amygdala lesions and improved by fornix lesions. Behav Brain Res 55: 269-281.

Yun IA, Fields HL (2003). Basolateral amygdala lesions impair both cue- and cocaine-induced reinstatement in animals trained on a discriminative stimulus task. Neuroscience 121: 747-757.

Zhuravin IA, Bures J (1991). Extent of the tetrodotoxin induced blockade examined by pupillary paralysis elicited by intracerebral injection of the drug. Exp Brain Res 83: 687-690. 